\title{
Two-stage Experimental Research Method for Fatigue Driving Based on Multiple Signal Sources
}

\author{
Xiaofan Wang ${ }^{a}$, Hongxu Chen, Xuyang Zhao ${ }^{\text {b, }}{ }^{*}$, Yuhao Wang and \\ Zhenhao Zhang
}

\author{
School of Automotive Engineering, Jilin University, Changchun, 130022, China. \\ aqyzxwxf0318@163.com, b, *1027473385@qq.com
}

Keywords: Fatigue driving, multiple signal sources, two-stage experiment.

\begin{abstract}
Fatigue driving is the main cause of traffic accidents. Through the study of driver behavior characteristics, indoor steering experiments were used to screen the steering wheel angle, grip force, accelerator pedal opening, pedaling frequency, and pulse frequency signal. The outdoor driving experiment was used to further screen the preliminary screening signals, and the behavioral characteristics were analyzed. The correlation between parameters and fatigue. The research on the fatigue monitoring of the driver using a variety of signals provides experimental basis and theoretical basis for the design of the driver's fatigue warning device.
\end{abstract}

\section{Introduction}

Traffic accidents have become more frequent in recent years. According to statistics from the World Health Organization, car accidents are ranked ninth in the causes of human death and morbidity. Among the many factors that cause car accidents, fatigue driving is one of the main factors that cannot be ignored. The US National Highway Traffic Safety Administration conservatively estimates that there are at least 100,000 traffic accidents caused by fatigue driving in the United States every year. In China, with the popularization of private cars, traffic accidents caused by fatigue driving are also increasing. More than $20 \%$ of traffic accidents are directly caused by fatigue driving. In fact, due to the fact that traffic accidents caused by driving fatigue are often difficult to define, it is actually much higher than $20 \%$. [1-2]

The detection of fatigue driving and the extraction of behavioral characteristics are the key issues in fatigue driving research. At present, the common detection and discrimination methods at home and abroad are mainly the following three types: The first type is a detection method based on the driving state of a vehicle, which determines a driver's mental driving state by measuring a plurality of parameters reflecting the running state of the vehicle, including a vehicle speed, an acceleration, and the like; and the second category is for the physiological and psychological characteristics of the driver. The detection method is mainly based on electroencephalogram (EEG), electrocardiogram (ECG) signals, facial features, etc. The third type is based on the detection of the driver's driving behavior, through the handling of steering wheel pressure changes, steering wheel angle signal processing by the driver.

In foreign countries, Sandberg measures a number of parameters that reflect the running status of the vehicle, including vehicle speed, acceleration, etc., and then establishes a BP neural network model to determine the driver's mental driving status. [3] Thump Chia Chief and others warn of driving fatigue and distraction by analyzing the pressure change of the driver's steering wheel. [4] Baulk finds that the driving ability of the driver. In particular, the lateral position of the vehicle has a good correlation with the reaction time. The reaction time can be used as an objective evaluation criterion for driving fatigue. [5]

In China, Wang Lianzhen used the driving environment attributes, driver's individual attributes, and original fatigue attributes as model input layer variables, and selected EEG, ECG, eye movement, and driving performance indicators as model output layer variables to be sober and light. Degree of fatigue and severe fatigue are three kinds of driver fatigue levels as hidden layer variables, and a 
driving fatigue degree recognition model based on Bayesian network is constructed. [6] Niue Inning et al. collected the steering wheel angle signal during the driving process through simulation experiments, and established a fatigue driving judgment model based on the steering wheel angle through the four-rotor feature extracted by the support vector machine. This kind of method can analyze the influence of each influencing factor on the fatigue driving behavior from the quantitative point of view, can reduce the monitoring cost, and provide a theoretical basis for carrying out the fatigue driving warning. [7]

Due to the driver's individual differences and the influence of the environment, the monitoring methods of a single physiological or behavioral indicator have great limitations and are prone to misjudgment and missed judgment. Therefore, people proposed a multi-source information fusion solution that applies multi-sensor fusion technology to driver fatigue monitoring. Multi-sensor fusion technology can not only improve the reliability and fault tolerance of the system, but also reduce the system cost, becoming an inevitable trend of fatigue driving detection.

In this experiment, the steering angle and angular velocity of the steering wheel were collected in real time by a 6-axis accelerometer equipped with a JY61 inclinometer. The four driving behavior signals, zero-speed percentage, average absolute value of rotation angle, standard deviation of rotation angle and angle entropy, were calculated; the steering wheel was real-timely acquired through the RFP602 piezoresistive film sensor. Grip signal; Pulse signal is obtained through the YK1303P heart rate sensor. By detecting the driver's reaction time to a specific stimulus as the basis for determining the degree of fatigue, the characteristic parameters that can determine the fatigue driving are found out, and the correlation with the fatigue degree is analyzed, which provides an experimental basis and a theoretical basis for the detection of fatigue driving.

\section{Experimental Design}

\subsection{Experimental Equipements and Acquired Signals.}

In the experiment, we collect the steering wheel angle and angular velocity in real time through accelerometer. After calculation, we get the data of zero-speed percentage, average absolute value of rotation angle, standard deviation of rotation angle, and angle entropy as driving signals. Besides, the grip force signal of steering wheel is collected by RFP602 pies resistive thin film sensor and the driver's pulse signal is obtained by YK1303P heart rate sensor. Thus, there are six signals identified as primary signals on behalf of driver's behavior.

\subsection{Experimental Process.}

This experiment adopts a combination of simulated experiment and driving experiment, which is a two-stage experimental research method. These signals were screened by the simulation experiment, and the driving test performed signal optimization. The overall flow chart was shown in Figure a.

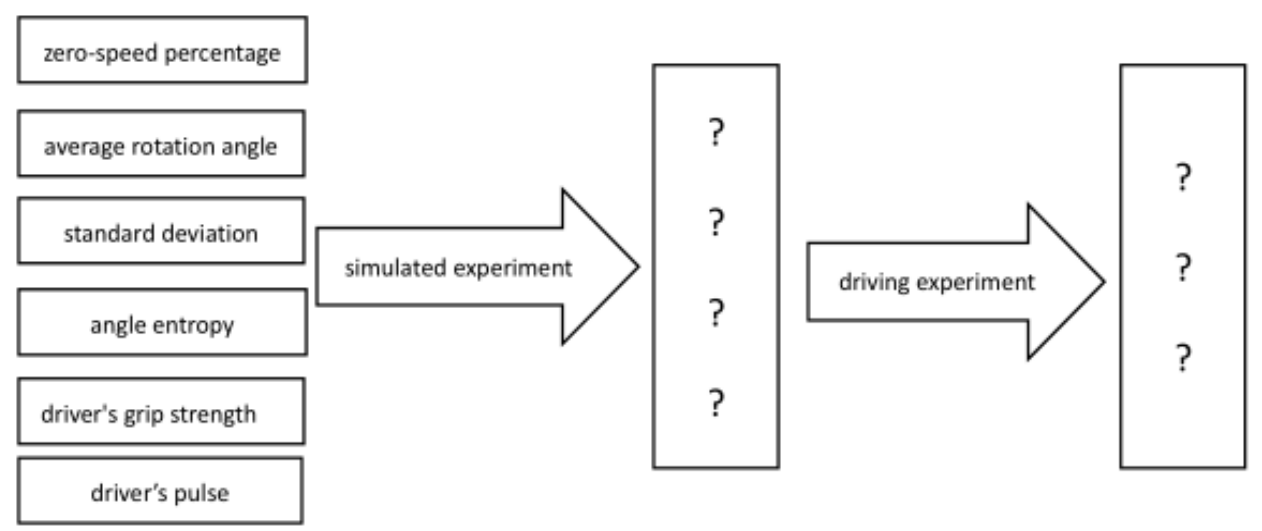

(a)

Figure. 1. The overall flow charts 


\section{Simulated Experiment}

\subsection{Equipment.}

The simulation experiment uses the Logitech G27 steering wheel pedal kit. It can help people feel the same as actual driving and can fully reflect the driver's behaviors. The device diagram is as follows.

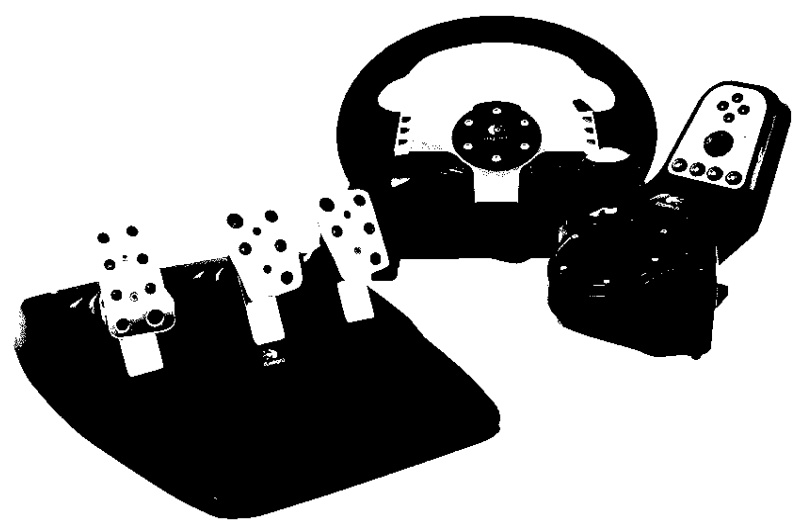

(b)

Figure. 2. Device diagram

\subsection{Experimental Arrangement}

We select five to ten non-professional drivers as subjects. In order to obtain more effective experimental data, a controlled experiment is conducted. Subjects are arranged to take part in the experiment at around 9:00 am and 1:00 pm after lunch. At 9 o'clock in the morning, the participants are required to ensure that they are fully awake and can be classified as normal driving groups. At one o'clock in the afternoon, the participants are required to ensure that they are in a state of complete fatigue and can be classified as a fatigue driving group.

In order to make up for the deficiencies caused by simulated driving experiments, we mainly chose a straight track and control the driving speed between 30 to $40 \mathrm{~km} / \mathrm{h}$ which is the normal speed of buses. Data starts collecting after 5 minutes of driving and is collected for a total of 10 minutes every time.

\section{Driving Experiment}

We select five to ten bus drivers who are in good condition as subjects. In order to obtain more effective experimental data, a controlled experiment is conducted. Subjects are arranged to take part in the experiment at around 9:00 am and 1:00 pm after lunch. The participants in the 9:00am are classified into normal driving groups. And the others are divided into a fatigue driving group. Before and after the experiment, these subjects are all required to fill in a subjective questionnaire of fatigue driving, and the data according with the grouping conditions are required as valid data. Otherwise, the subjects are required to participate in the experiment another day.

People's Avenue in Changchun City is chosen as experimental rode. The road has a total length of $13.7 \mathrm{~km}$, a width of $54 \mathrm{~m}$, with a smooth road surface. In the experiment, copilot assists to ensure driving safety, the speed of the bus was about $30-40 \mathrm{~km} / \mathrm{h}$, and data is collected after 5 minutes of driving. Data has been collecting for 10 minutes. The data collected during normal driving on a straight road is valid data.

The aim of driving experiment is to optimize the driver's fatigue driving signal after the preliminary signals of the simulated driving experiment, so as to acquire a more accurate experimental conclusion.

\section{Fatigue Detection}

In order to mark the fatigue degree of the test, at the beginning and the end of driving, there should be a fatigue detection experiment through calculating the time of the visual reaction. Subjects need to 
turn off the switch after the light is on, to measure the response time of the test during the test period. The time from the beginning of the lamp to the moment the lamp is turned off (the lamp-on time) is denoted as $\mathrm{T}$. According to the size of $\mathrm{T}$, we can divide fatigue into three levels that is soberness, mild fatigue and severe fatigue.

The subjective questionnaire of fatigue driving is shown in Figure c.

\begin{tabular}{|c|l|l|l|}
\hline Name & & Sex & Age \\
\hline Experiment Time & ( ) 9:00 am & ( ) $1: 00 \mathrm{pm}$ \\
\hline Experiment Type & ( ) Simulation Experiment $\quad$ ( ) Driving Experiment \\
\hline $\begin{array}{c}\text { Experiment Group } \\
\text { ( ) Normal Driving }\end{array}$ & ( ) Fatigue Driving \\
\hline $\begin{array}{c}\text { Pre-experimental } \\
\text { Degree }\end{array}$ & ( ) Soberness & ( )Mild Fatigue & ( ) Serve Fatigue \\
\hline $\begin{array}{c}\text { Post-experimental } \\
\text { Degree }\end{array}$ & ( ) Soberness & ( )Mild Fatigue & ( ) Serve Fatigue \\
\hline
\end{tabular}

(c)

Figure. 3. The time from the beginning of the lamp

\section{Conclusion}

The experimental detection system has little influence on the driver's operation which means a high recognition accuracy in the experiment. At the same time, the two-stage experiment method is used to analyze the correlation between various characteristic parameters of the driver and the fatigue driving, so the accuracy is improved. The research provides the experimental basis and theoretical basis for the detection of fatigue driving.

\section{References}

[1]. Wu Dequan. Hazard and Prevention of Fatigue Driving [J]. Science \& Technology Outlook, 2015(35).

[2]. Wu Chunking. Discussion on Research Progress of Automobile Driver Fatigue Monitoring Technology [J]. Mechatronics, 2007, 13 (4): 43-47.

[3]. Sandberg D. Analysis and optimization of systems for detecting sleepless in drivers. GO tabor: Chalmers University of Technology, 2008.

[4]. Thump Chia chief Mustafa, Hussain M, Hazed A, et al. Driver fatigue detection using steering grip force [C] // Malaysia, IEEE: Student Conference on Research and Development, Malaysia: IEEE 2003:45-48.

[5]. Baulk S Debugs S Nereids K Jet al. chasing the silver bullet: measuring driver fatigue using simple and complex tasks [J]. Accident Analysis and Prevention.2008, 40(1):396-402.

[6]. Niue Inning. Research on fatigue driving detection method based on information fusion [D]. Changchun: Jilin University, 2014.

[7]. Wang Lianzhen, Pei Young. A Recognition Model of Driver's Fatigue Based on Bayesian Network [J]. Urban Transport, 2014, 03:66-74. 